\title{
Multi-layer HIS of Circular Protrusions; Design \& Analysis
}

\author{
K.Praveen Kumar \&Dr Habibulla Khan \\ ${ }^{1}$ Associate Professor, Dept of ECE, MLRIT, JNT University Hyderabad, Telangana, \\ India \\ ${ }^{2}$ Professor, Dept of ECE, KL University Guntur, Andhra Pradesh, India
}

\begin{abstract}
In this paper two types of multi-layer mushroom type high impedance surfaces (HIS) with a protrusions of shape circular, prepared with PEC material are investigated. The architecture of first model consists of arrangement of circular patches in a square array and second model is prepared with a triangular array of circular protrusions. Both the architectures consists of upper and lower layers of protrusions. The reflection phase characteristics of above two structures are studied, In multi-layer environment the effect of some parameters on the reflection phase characteristic is discussed.
\end{abstract}

\section{KEYWORDS}

HIS, Reflection phase, Thickness, Operating frequency, surface wave(SW)

\section{INTRODUCTION}

The unique characteristics of high impedance surfaces are forbidding the SW and zero-phase reflection for plane waves made these structures applicable in many antenna and microwave applications[1-3]. The first property of surface wave suppression improves efficiency, produce smoother radiation pattern, and exhibit less back lobe and side lobe levels when embedded within millimeter wave applications [1,2]. On the other hand, these structures facilities low profile antennas operate adjacent to the them without getting shorted $[1,3]$ is another advantage.

The reason behind considering of multi-layer high impedance surface in present paper is to obtain lower operating frequency or in phase reflection frequency when compared with two dimensional EBG structures. This property can possible to prove when we computed the reflection phase characteristics of multi layer HIS structure under normal incident plane wave illumination. The wire antenna placed over this multi layer HIS must operate within the input match frequency region. where input match frequency region can be defined as " the region with in which the HIS reflection phase characteristics are in phase" this explained in [3].So the region between +45 degree to -135 degree of a reflection phase curve is considered as very close to input-match frequency of stop band. Where low profile wire antenna is made to operate so that to obtain enhanced characteristics. The antenna which is operating with in this region can place directly adjacent to the high impedance surface without getting shorted out. Hence reflection 
phase of proposed HIS periodic structure is computed ,by considering an unit cell. The complete design process is presented in[4].

In present paper we show that a multi-layer HIS has significantly lower zero-reflection phase frequency when compared with a 2D HIS and after that we investigate the effect of the multilayer structures by considering t's reflection phase characteristics. By means of these curves, we can suggest a design guidelines, that make easier in designing of multi-layer mushroom structures, which can be effectively used as ground plane low profile antennas. Finally, three different configurations of multi-layer HIS are compared based on their reflection phase characteristics.

\section{MULTI LAYER CIRCULAR SHAPED HIS}

Figure 1 showing two types of multi-layer protrusions of circular shaped high impedance surfaces. The patches in both the upper layer and lower layers has a radius $r_{t}$ and $r_{b}$, respectively. In present proposal the value of $r_{t}$ is made equal to $r_{b}$. and the gap $g$ which is $0.5 \mathrm{~mm}$ is maintained between patches.The Fig. 1(a) is depicting the arrangement of circular protrusions in multi layer are located so as to forma square array while Fig. 1(b) is showing triangular array arrangement of circular patches. $\varepsilon_{r 1}$ and $\varepsilon_{r 2}$ are the substrate permittivity of the upper and lower layers respectively. The upper layer thickness is considered as $t$ and lower layer thickness is $h$.

Table 1: 2D-HIS and Multi layer HIS design parameters.

\begin{tabular}{|c|c|c|}
\hline Parameter & Value & Units \\
\hline $\mathrm{t}$ & 0.1 & $\mathrm{Mm}$ \\
\hline $\mathrm{h}$ & 3 & $\mathrm{Mm}$ \\
\hline$\varepsilon_{\mathrm{r} 1}$ & 3.25 & \\
\hline$\varepsilon_{\mathrm{r} 2}$ & 4.4 & $\mathrm{Mm}$ \\
\hline $\mathrm{r}$ & 4 & $\mathrm{Mm}$ \\
\hline $\mathrm{g}$ & 0.5 & \\
\hline
\end{tabular}

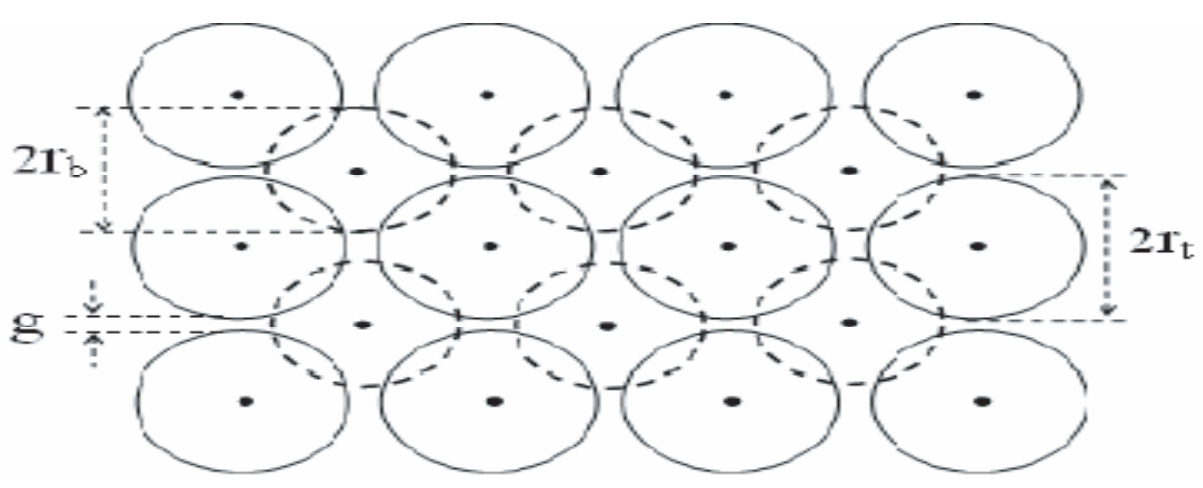


(a)

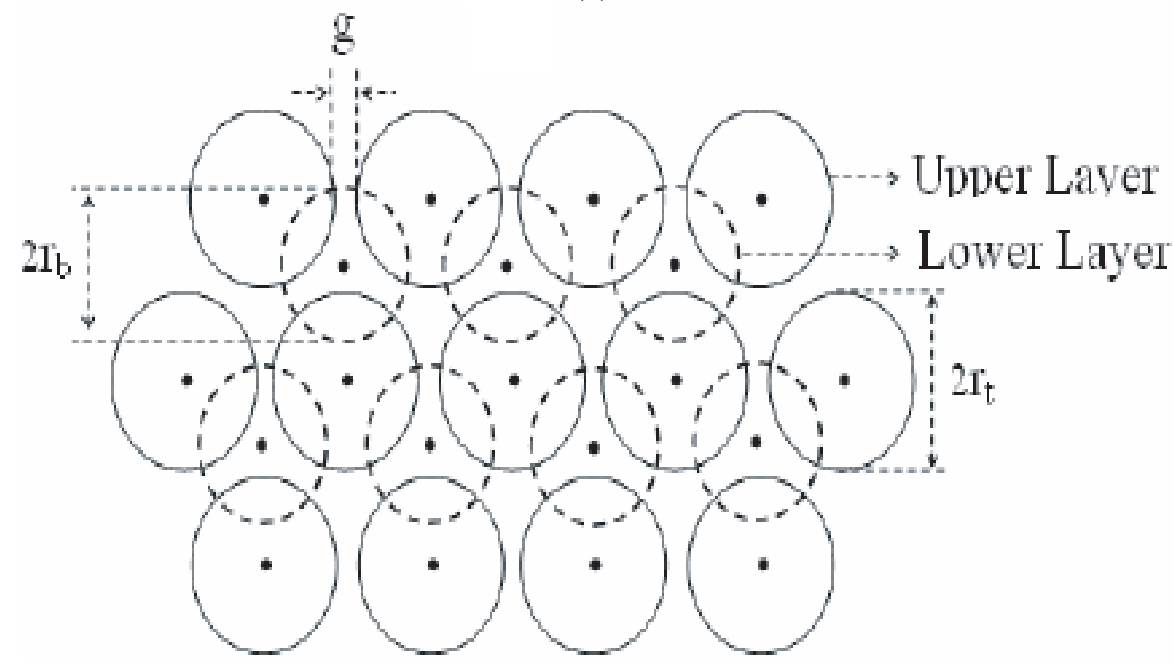

(b)

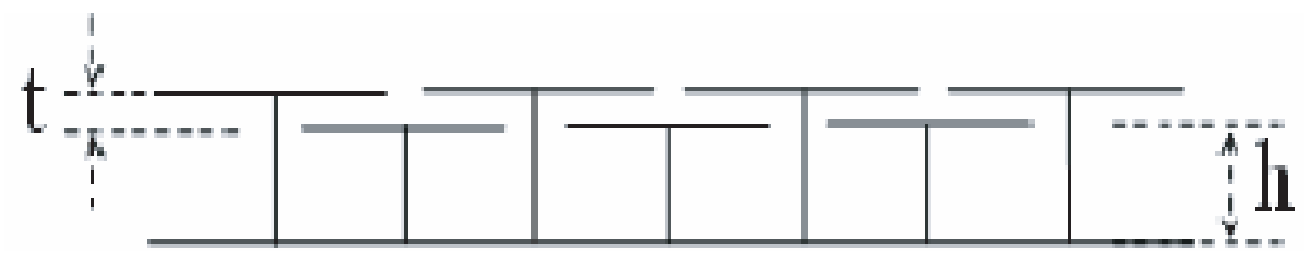

(c)

Figure 1: Multi-Layer Mushroom-Like HIS: (A) Circular Protrusions Of Square Array, (B) Circular Protrusions Of Triangular Array, (C) The Side View Of Multi Layer HIS

A plane waves which is incident normal to the high impedance surface is considered in order to measure the reflection phase characteristics of a HIS. The concept here is presented in three stages. During first stage, the reflection phase characteristics of a 2D HIS with circular protrusions is designed and computed so as to obtain result,which was depicted in Fig. 2(a). In this model the circular patches having radius $r$ are printed on the top side of substrate layer which has a height of $t+h$.Another side of substrate contains ground plane. The design parameters and it's values are listed in Table 1. It's zero reflection phase frequency is around 6GHz.The figure 1 is showing the multi layer HIS whose reflection phase characteristics are depicted in figure. 2(b). The zero-reflection phase frequencies of both multi-layer HISs are at $1.6 \mathrm{GHz}$ and $1.8 \mathrm{GHz}$ respectively. These values are much lower than that of the 2D HIS. Also, the multi layer HIS contains triangular array of circular protrusions has lower zero-reflection phase frequency when compared with multi layer HIS contains square array of circular protrusions. 
International Journal of Microwave Engineering (JMICRO) Vol.1, No.1, January 2016

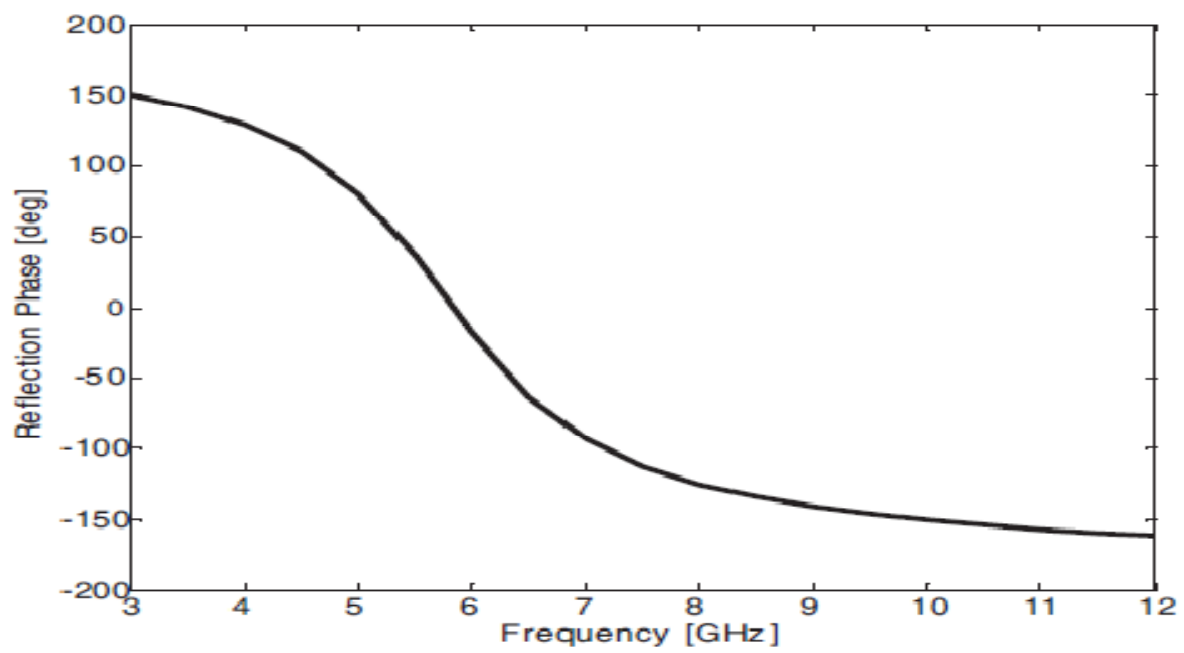

Figure 2 (A) 2D-HIS Reflection Phase Characteristic

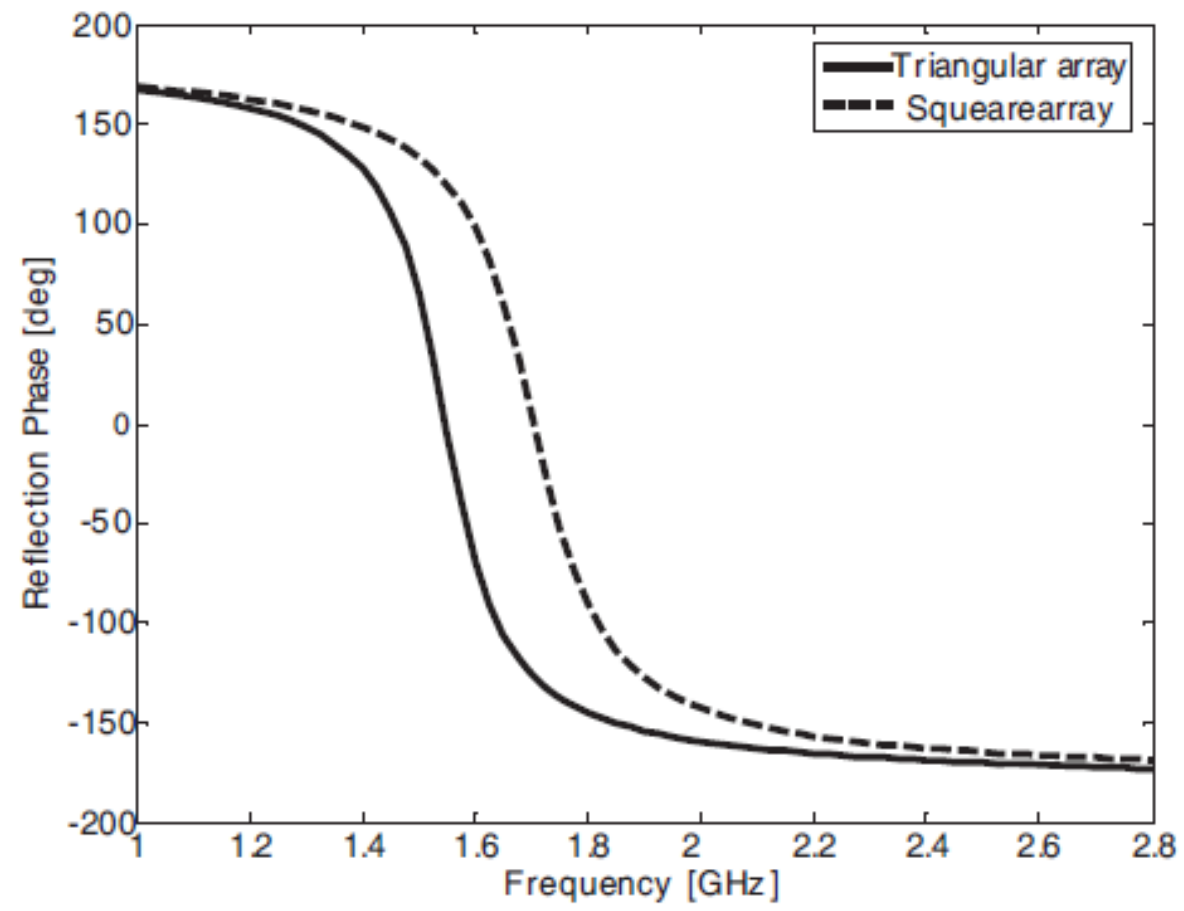

Figure 2 (B) Multi Layer HIS Reflection Phase Characteristics For Two Cases.

\section{PARAMETRIC STUDY}

The main motive behind the parametric study is to understand behavior of proposed structure. The parametric analysis of 2D HIS presented in[3]. Similar process is applied to present structure. Amulti-layer HIScontains four key parameters they are thickness(t), height(h), radius(r) and $\operatorname{gap}(\mathrm{g})$. show that, the zero reflection phase frequency would decreased in both the 2D-HIS and multi layer HISwhen a dielectric constant and radius of patches or thickness of the lower layer increases and when decrease in gap. 
International Journal of Microwave Engineering (JMICRO) Vol.1, No.1, January 2016

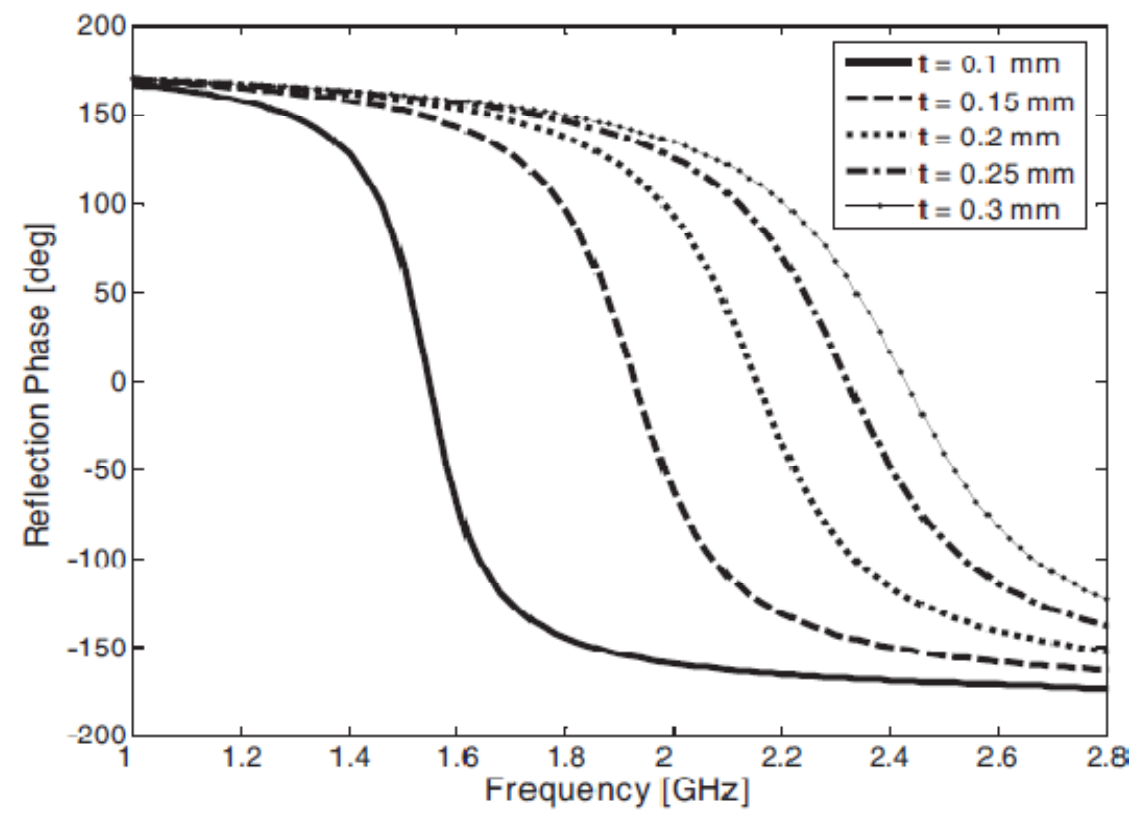

Figure 3: Effect of the Upper Layer Thickness on the Reflection Phase Characteristic.

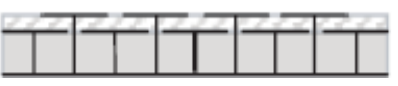

(a)

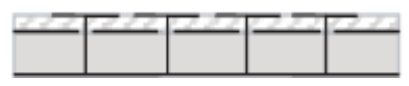

(b)

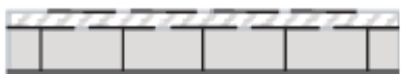

(c)

Figure 4: EBG Surfaces With Three Different Configurations.

The figure 3 is depicting the parametric study of multi layer HIS of circular protrusions are arranged in triangular array.The substrate thickness $t$ is considered here for analysis. To analyze a via effect in multi layer HIS design here three cases are considered which are shown in figure 4. The reflection phase characteristics for three cases were presented in figure 5. The reflection phase characteristics obtained in three casses are similar hence in order to reduce the complexity and manufacturing cost it is preferable to consider figure 4(b) and (c) instead of (a)

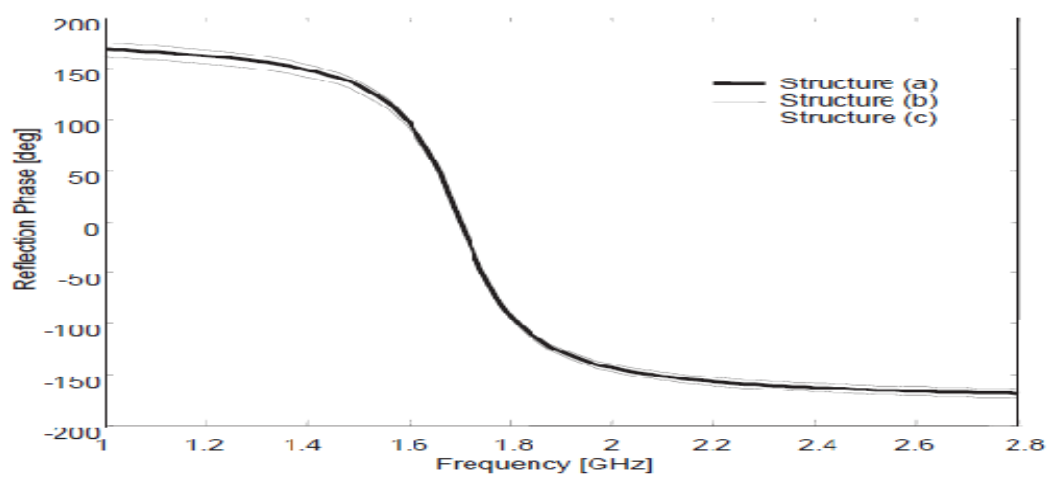

Figure 5: Via Effect Analysis 


\section{CONCLUSION}

Present paper two architectures of multi layer HIS designed and its reflection phase characteristics studied in comparison with conventional 2D-HIS.The similarities in parametric study in both multi layer HIS and 2D HIS is presented. In order to reduce the manufacturing complexity the via effect is analyzed and proposed simple and effective model.

\section{REFERENCES}

1. Engheta, N. and R. W. Ziolkowski, Metamaterials Physics and Engineering Explorations, Wiley, New York, 2006.

2. Sievenpiper, D., L. Zhang, R. F. J. Broas, N. G. Alexopolus, and E. Yablonovitch, LHigh- impedance electromagnetic surfaces with a forbidden frequency band," IEEE Trans. Microwave Theory Tech., Vol. 47, 2059-2074, Nov. 1999.

3. Yang, F. and Y. Rahmat-Samii, "Reflection phase characterizations of the EBG ground plane for low profile wire antenna applications," IEEE Trans. Antennas Propag., Vol. 51, 2691-2703, Oct. 2003.

4. Remski, R., "Analysis of PBG surfaces using Ansoft HFSS," Microwave J., Vol. 43, No. 9, 190-198, Sept. 2000.

\section{AUTHORS}

K.Praveen Kumar Associate Professor, was born in india, A.P in 1980 . He receivedB.E(ECE) from Visveswaraiah Technological University, Belgaum. AndM.Tech(Microwave Engg) Acharya Nagarjuna University, Guntur. He has more than 10years of teaching experience. 14 International Journals, 03 International Conference in hiscredit. $\mathrm{He}$ is a research scholar of JNT

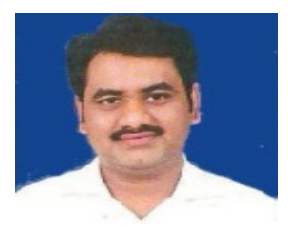
University, Hyderabad. In the field of MicrowaveAntenna.

Prof. Habibulla khan born in India, 1962. He obtained his B.E. from V R Siddhartha Engineering College, Vijayawada during 1980-84. M.E from C.I.T, Coimbatore during 1985-87 and PhD from Andhra University in the area of antennas in the year 2007.He is having more than 20 years of teaching experience and having more than 20 international, national journals/conference papers in his credit.Prof. Habibulla khan presently

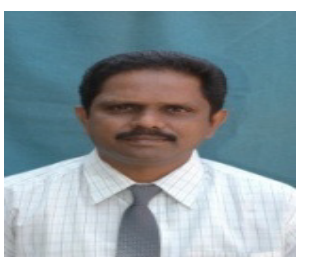
working as Head of the ECE department at K.L.University. He is a fellow of I.E.T.E, Member IE and other bodies like ISTE. His research interested areas includes Antenna system designing, microwave engineering, Electro magnetics and RF system designing. 